DESCRIPCION BASICA DEL PINO OREGON (Pseudotsuga menziesii). Ute Kannegiesser Schuh, Ingeniero Forestal, División Regional, Instituto Forestal. Barros Arana 121. ConcepciónChile.

\title{
INTRODUCCION
}

Chile cuenta con un vasto potencial de recursos forestales. De aproximadamente 9 millones de hectáreas de bosques productivos, un $14 \%$ corresponde a plantaciones artificiales con especie extóticas. Entre éstas la de mayor importancia es Pinus radiata, con una superficie de 1.118 .088 ha a Diciembre de 1987 (CORFO - INFOR, 1988). La producción forestal chilena está basada en esta especie. Indicios claros de ataques ocasionados por agentes patógenos en algunas áreas, la marginalidad de desarrollo para esta especie en muchos sectores y la necesidad de impulsar una oferta de productos forestales más variada, han llevado al INSTITUTO FORESTAL a la investigación de otras especies de rápido crecimiento y buenas perspectivas económicas.

Una conifera con atractivas proyecciones para nuestro pais es Pseudotsuga menziesii. Esta especie es de origen norteamericano. Su rápido crecimiento, las características de su madera y su adaptabilidad a una gran variedad de ambientes han sido las causas de su introducción en muchos países en el mundo. Hasta fines del año 1987, las plantaciones en Chile con esta especie cubrian aproximadamente 11.000 ha entre la VIII y XI Regiones. Su rendimiento según el sitio varia entre 15 y $30 \mathrm{~m}^{3} / \mathrm{ha}$ al año.

El objetivo de este trabajo es entregar antecedentes generales sobre la especie Pino Oregón (Pseudotsuga menziesii), haciendo referencia a temas como su distribución, sus requerimientos en cuanto a clima, suelos, las asociaciones vegetales a que pertenecen y su importancia económica.

\section{GENERO PSEUDOTSUGA}

El género Pseudotsuga está compuesto por un pequeño grupo de coníferas arbóreas siempreverdes, caracterizadas por su longevidad y las grandes dimensiones que alcanzan en su hábitat natural. En la costa oeste de Norteamérica existen ejemplares que, además de algunos del género Sequoia, son considerados las coniferas más grandes del mundo (BAILEY, 1960; STREETS), 1962; LITTLE, 1979). Su distribución natural abarca el Hemisferio Norte y actualmente está restringido al oeste de Norteamérica y al este de Asia (HERMANN, 1985). Los representantes asiáticos del género Pseudotsuga se encuentran aislados en regiones montañosas al suroeste y este de China, sur y centro de Japón y en Taiwán.

Desde el punto de vista económico, las especies norteamericanas son consideradas las más importantes y son las que preferentemente se han introducido a nivel mundial. Entre éstas destaca Pseudotsuga menziesii por su gran valor maderero y las existencias en pie (DALLIMORE y JACKSON, 1961; BURSCHEL y HUSS, 1987).

Actualmente el nombre Pseudotsuga menziesii (Mirb.) Franco ha sido aceptado por la gran mayoría de los botánicos, aunque en Europa todavía se utilizan los sinónimos Pseudotsuga taxifolia (Poir.) Britton y Ps. douglasii (D. Don). Esta especie se conoce con los nombres vulgares como Douglas-fir, abeto de Douglas, sapin de Douglas (franc.) y Douglastanne (alem.) (BAILEY, 1969; HOSIE, 1969; HERMANN, 1982). En Chile, se conoce con el nombre de pino oregón. Comercialmente se denomina "Oregon pine", "Yellow o Red fir" (HOSIE, 1969; USFPL 1974; HERMANN, 1982). 


\section{Descripción de la especie Pseudotsuga menziesii}

En Norteamérica Pseudotsuga menziesii es un árbol forestal de grandes dimensiones. Generalmente alcanza 50 a $60 \mathrm{~m}$ y, ocasionalmente, $90 \mathrm{~m}$ de altura y diámetros a la altura del pecho entre 2 y 4,5 m (DALLIMORE y JACKSON, 1961; HOSIE, 1969; BROCKMANN, 1979; LITTLE, 1979). El fuste recto, cilíndrico y ligeramente cónico está coronado por una copa piramidal con ramas horizontales dispuestas en verticilos. En árboles jóvenes, la copa llega hasta el suelo.

La corteza es de color gris, delgada y se encuentra cubierta por bolsas de resina. A medida que el árbol envejece, se torna café-rojiza, gruesa, esponjosa y agrietada. Contiene alrededor de 20 a $50 \%$ de tejido suberoso y aproximadamente un 30\% de extraíbles como son ceras, caninas y otras sustancias químicas (ISAAC y DIMOCK, 1958).

Las acículas son de color verde-amarillento o azulado, lineales y planas. Se angostan hacia la base, formando un pecíolo corto. En la cara superior presentan dos bandas estomáticas separadas por la vena media y, por el envés, 2 canales resiníferos superficiales. El follaje, especialmente molido, despide un fuerte olor a trementina (DALLIMORE y JACKSON, 1961).

Pseudotsuga menziesii se caracteriza por los conos péndulos de color café con escamas tectrices exertas y trífidas (Fig. 1). Bajo las brácteas ovulíferas se ocultan 2 semillas café-rojizas.

\section{FIGURA 1}

\section{CONO CON ESCAMA EXERTA Y TRIFIDA (a) Y HOJAS (b) DE PSEUDOTSUGA MENZIESII}

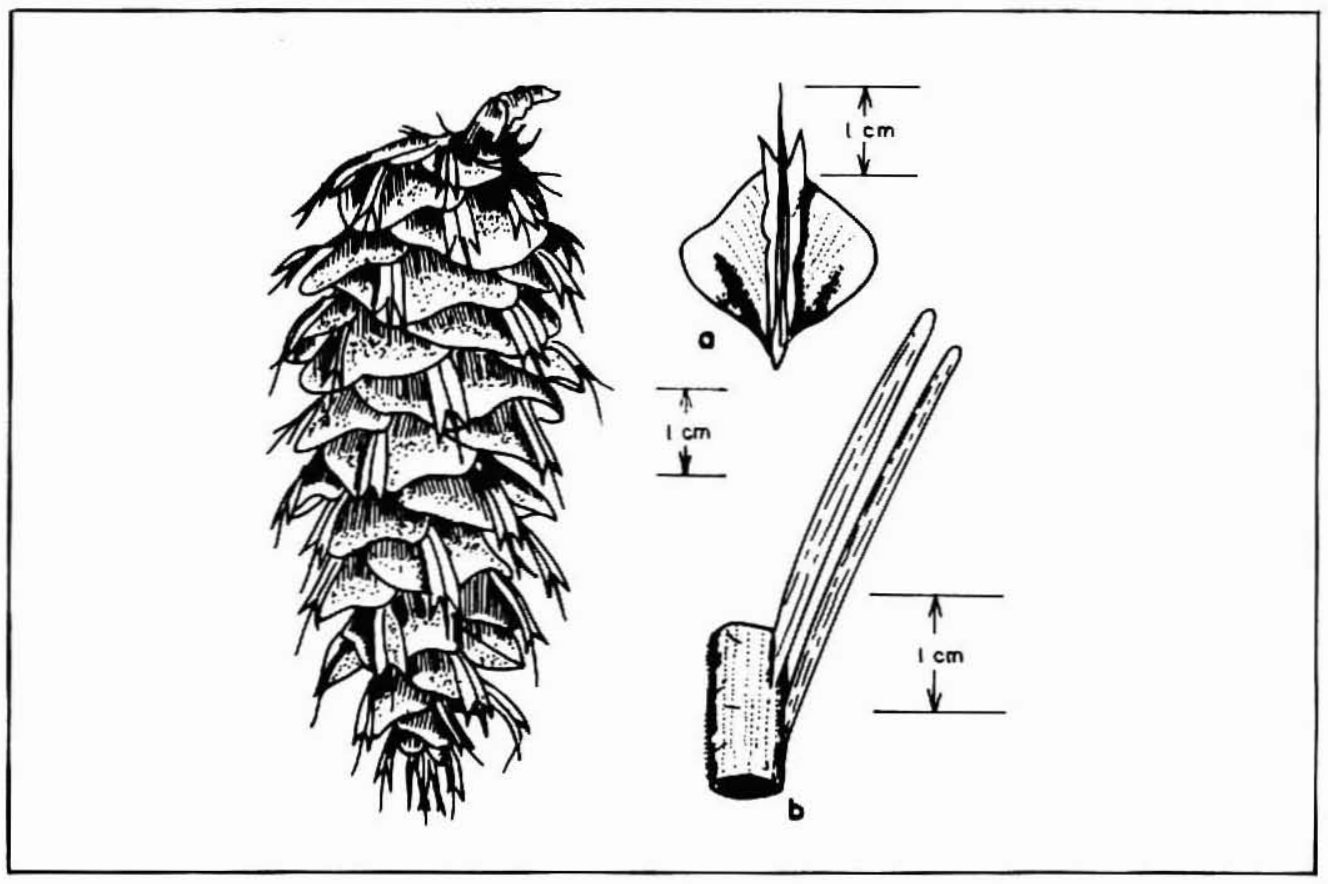

Fuente: RODRIGUEZ y RODRIGUEZ, 1981. 
En su habitat natural se obtienen trozas de grandes dimensiones libres dé nudos; La madera de pino oregón norteamericano es dura, resistente, pesada, de grano recto y textura fina. Se caracteriza por ser de gran resistencia mecánica. Además es fácil de trabajar y acepta bien pinturas y barnices. Los anillos de crecimiento están claramente definidos. La albura es de color blanco-amarillento. Arboles con crecimiento diametral rápido forman un duramen de color café-rojizo y textura gruesa. En cambio, en etapas de crecimiento más lento, el duramen es de color café-amarillento.

El duramen es resistente a la pudrición y a la acción de ácidos orgánicos y minerales diluidos. Presenta resistencia a la penetración de agua, lo que dificulta la inyección de cualquier preservante. En EE.UU. se estima una vida últil de 6 a 9 años para durmientos sin tratamientos (USFPL, 1974).

Todas estas características hacen que la madera de Pino Oregón sea muy apropiada para su uso en elementos estructurales, obras mayores de construcción como puentes, viviendas en general, y embarcaciones. La madera también es usada en la industria de chapas, tableros, madera aserrada y elaborada, marcos de puertas y ventanas, embalaje, toneleria, durmientos, pallets, cajas y cajones, acueductos, revestimientos de interior y exterior, postes, pilotes, rodrigones, ceras y pulpas.

A pesar de que no existen bases taxonómicas para segregar otras variedades, algunos botánicos europeos reconocen una tercera. SCHENK $(1939$, citado por FOERST, 1980) divide la especie en viridis o verde, glauca o azul y caesia o gris, delimitando sus distribuciones en función de zonas climáticas de crecimiento. En el sector norte de las Montañas Rocallosas se encuentra la variedad caesia y en la parte sur, la glauca. Pseudotsuga menziesii var. menziesii o viridis, ocupa la región entre la cadena montañosa y el Océano Pacífico.

En Norteamérica se diferencian dos variedades Pseudotsuga menziesï (Mirb.) Franco var. menziesii o costera y Pseudotsuga menziesii var. glauca (Beissner) Franco o interior. Ambas difieren en el color del follaje, la forma del cono, la tasa de crecimiento y los requerimientos ambientales. El término costero ha causado problemas porque la variedad se extiende considerablemente hacia el interior del territorio, alejándose de la costa.

El pino oregón costero (Pseudotsuga menziesii var. menziesii) es el más importante dentro de las variedades. Tanto en su pais de origen como en los países donde se ha introducido es considerado una especie interesante económicamente por su crecimiento rápido, buena forma, sanidad y la calidad de su madera.

\section{Distribución natural}

La especie Pseudotsuga menziesii se distribuye desde los $55^{\circ} \mathrm{LN}$ hasta $\operatorname{los} 20^{\circ} \mathrm{LN}$ en el oeste de América del Norte (BURSCHEL y HUSS, 1987). Hasta aproximadamente los $36^{\circ} \mathrm{LN}$ se extiende en forma continua entre la costa del Pacífico y la ladera este de las Montañas Rocallosas. Hacia el sur, se aleja de la costa permaneciendo en los sectores más montañosos hasta alcanzar su limite al sur de México.

Una distribución más restringida presenta la variedad menziesii, extendiéndose a lo largo de la costa del Pacífico desde el estado de Columbia Británica $\left(53^{\circ} 30^{\prime} \mathrm{LN}\right)$ a través de los de Washington y Oregon hasta el de California central $\left(36^{\circ} \mathrm{LN}\right)$. Su límite oriental lo forman las altas cumbres de las Cascadas y la Sierra Nevada (Fig. 2).

En relación a las altitudes, esta variedad cubre territorios desde el nivel del mar hasta la cima de la cordillera de las Cascadas. En el extremo norte de su distribución alcanza su límite altitudinal aproximadamente a los $800 \mathrm{~m} . \mathrm{s} . \mathrm{n} . \mathrm{m}$.

A medida que se desplaza hacia el sur, el límite de distribución en altura aumenta, llegando hasta 1.800 m.s.n.m. en la frontera norte del estado de California $\left(42^{\circ} \mathrm{LN}\right)$ (FOWELLS, 1965; ISAAC y DIMOCK, 1958). 
FIGURA 2

DISTRIBUCION NATURAL DE PINO OREGON COSTERO (PSEUDOTSUGA MENZIESII VAR. MENZIESII)

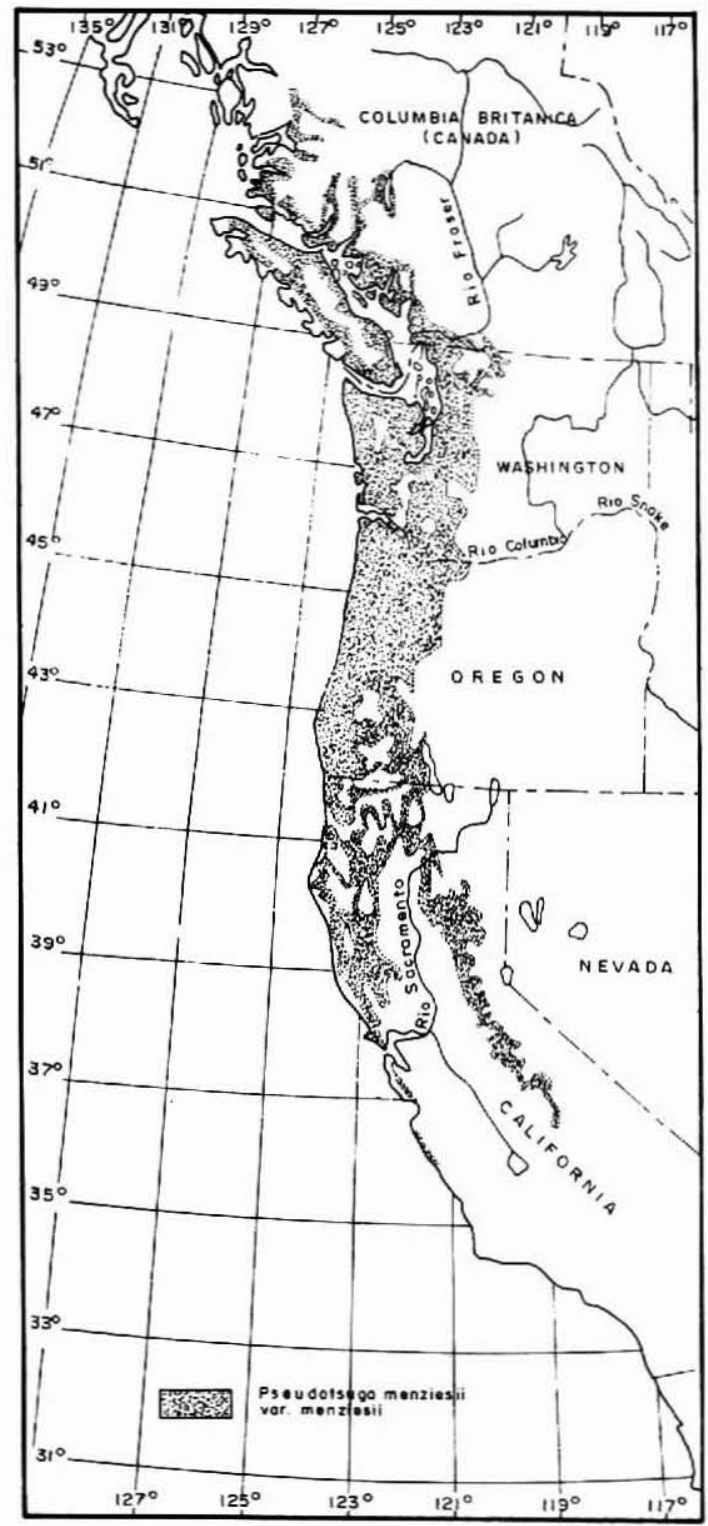

Fuente: ISAAC y DIMOCK, 1958. 


\section{Clima}

El área de distribución natural de Pseudotsuga menziesii var. menziesii presenta un clima templado y húmedo, que favorece el crecimiento de las coníferas.

Las precipitaciones son de régimen invernal, cayendo sólo el $30 \%$ durante la primavera y el verano. Este porcentaje aumenta considerablemente si se incluye la influencia oceánica que existe en el sector, manifestada en forma de frecuentes neblinas. Los niveles pluviométricos anuales fluctúan entre 500 y $2.300 \mathrm{~mm}$, con un promedio de $1.400 \mathrm{~mm}$ (ISAAC y DIMOCK, 1958). Durante el verano la humedad relativa oscila entre 50 y $60 \%$.

La temperatura media anual varía entre $7^{\circ}$ y $13^{\circ} \mathrm{C}$ con valores máximos y mínimos absolutos de 43 y $-34^{\circ} \mathrm{C}$ respectivamente. La influencia oceánica disminuye hacia el interior del continente. Esto explica el aumento de los días con heladas en los sectores más montañosos. En la costa se observan en promedio 212 días libres de heladas, comparado con 138 en las montañas (FOWELLS, 1965).

\section{Suelos}

En el área de distribución natural de Pseudotsuga menziesii var. menziesii se presentan los siguientes grupos de suelos: Pardos, Pardos podzólicos, Pardos lateríticos, Latosoles pardos y Pardoforestales. Entre la cordillera de la Costa y el Océano Pacífico, los suelos son principalmente de origen sedimentario con intrusiones de rocas volcánicas. En cambio, al este de la cordillera de la Costa hasta la cima de las Cascadas, el origen del suelo es volcánico (ISAAC y DIMOCK, 1958).

El Pino Oregón costero crece en un amplio rango de tecturas, variando desde arcillas gravosas hasta arenas arcillosas (FOWELLS, 1965). Se desarrolla en tipos de suelos ácidos, con valores de $\mathrm{pH}$ que fluctúan entre 4,5 y 6,5. En el oeste de Washington y Oregon alcanza sus mejores desarrollos en suelos con pH 5 a 5,5. La mejor ocupación del sitio y los mayores volúmenes los logra cuando se encuentra en suelos arcillosos profundos, con buena retención de agua, pero bien drenados.

No prospera en suelos mal drenados, pantanosos y deficientes en oxígeno, como tampoco en aquellos que presentan "hardpans" superficiales. Esta capa impide el buen desarrollo de su sistema radicular y un drenaje adecuado (ISAAC y DIMOCK, 1958; FOWELLS, 1965).

\section{Asociaciones vegetales}

El Pino Oregón se distribuye a través de un amplio rango geográfico y así está sometido a diferentes condiciones climáticas. Debido a esto, a lo largo de su distribución se encuentra asociado con distintas especies arbóreas y arbustivas.

En el límite norte por la costa, Pseudotsuga menziesii var. menziesii crece con Tsuga heterophylla. Thuja plicata y Picea sitchensis. A medida que este bosque se acerca a su estado climax, el Pino Oregón sólo queda representado por algunos individuos aislados en el estrato arbóreo dominante.

Hacia el sur de su rango de distribución se encuentra acompañando a Sequoia sempervirens, que lo desplaza gradualmente. También se mezcla con Pinus lambertiana, Pinus ponderosa, Libocedrus decurrens y varias encinas (Quercus spp).

A mayor altitud, hacia las Montañas Rocallosas el Pseudotsuga menziesii var. menziesii es reemplazado por su variedad glauca, por especies del género Abies y Tsuga mertensiana. 
En los sectores más secos de su distribución, la variedad menziesii forma rodales puros y coetáneos. En aquellos más húmedos crece asociado con especies latifoliadas como Alnus rubra, Arbutus menziesii y algunas del género Acer.

FOWELLS (1965) describe los principales tipos forestales, donde Pseudotsuga menziesii var. menziesii es una especie dominante:

1. Pacific Douglas-fir

2. Pseudotsuga menziesii - Tsuga heterophylla

3. Chamaescyparis lawsoniana-Ps. menziesii

4. Pinus ponderosa - Ps. menziesii

Además es una especie acompañante en las siguientes asociaciones:

$\begin{array}{ll}\text { 1. } & \text { Alnus rubra } \\ \text { 2. } & \text { Picea sitchensis } \\ \text { 3. } & \text { Abies amabilis }- \text { Tsuga } \mathrm{sp} \\ \text { 4. } & \text { Thuja plicata }- \text { Tsuga heterophylla } \\ \text { 5. } & \text { Sequoia sempervirens } \\ \text { 6. Quercus alba } \\ \text { 7. }\end{array}$

\section{Importancia económica}

Pseudotsuga menziesii es una de las especies forestales de mayor importancia en Norteamérica. El inventario forestal de 1977 establece que esta especie ocupa 12,5.millones de ha, lo que equivale al $6,4 \%$ de la superficie total de bosques comerciales que EE.UU. (USDA, 1982). Se estima un volumen neto de 2.648 millones de $\mathrm{m}^{3}$ sin corteza, del cual aproximadamente el $60 \%$ se encuentra en árboles con diámetros a la altura del pecho, mayores de $48 \mathrm{~cm}$.

En estos bosques existe el $8 \%$ de las reservas totales de madera de los EE.UU., el $21 \%$ de aquellas de madera aserrable y el $28 \%$ del volumen total contenido en árboles con diámetros mayores de $48 \mathrm{~cm}$ (SUTTON, 1978).

En Canadá, el Pino Oregón está representado solamente en el estado de Columbia Británica, existiendo 721 millones de $\mathrm{m}^{3}$ con corteza (UNECE - FAOEFC, 1985).

Esto corresponde al 3\% del volumen total de madera de Canadá y al $7 \%$ de Columbia Británica. Debido al fácil acceso, a la alta calidad de estos bosques y a la existencia de poderes compradores, actualmente el Pino Oregón es la especie forestal más explotada, con una participación del $4 \%$ en el volumen total de corta anual (SUTTON, 1978).

Esta especie ha sido plantada en muchos parques y jardines con fines ornamentales. Su sistema radicular bien desarrollado le da estabilidad, por lo que es considerada apta como cortina cortavientos.

La alta calidad de la madera, la producción de grandes volúmenes comerciales, su fácil propagación por semilla, su rápido crecimiento $\mathrm{y}$, en general, la plasticidad frente a factores ambientales han incentivado la introducción de Pino Oregón en distintos paises a nivel mundial. 
En Europa central actualmente es la especie introducida de mayor importancia. Desde hace tres décadas las superficies forestales con Pino Oregón están aumentando en la República Federal Alemana (BURSCHEL y HUSS, 1987). Aproximadamente 100 años después del inicio de las plantaciones con Pino Oregón en el suroeste de Alemania ya existen 7.000 ha de bosques estatales (SCHÜL, 1984). Se estima que en ese país llegará a ser la segunda conífera más importante después de Picea sp, desplazando a Pinus spp (BURSCHEL y HUSS, 1987).

La principal especie de reforestación en Francia es Pino Oregón. Cada año se plantan entre 10 y 15 mil hectáreas de un total de 40 a 50 mil. Actualmente se estima que existen 220.000 ha, compuestas principalmente por plantaciones menores de 25 años (OSWALD y PARDE, 1984). En Alemania y Francia se prefiere la variedad menziesii. Especialmente por problemas fitosanitarios, se prohibió la introducción de la variedad glauca en Alemania (BURSCHEL y HUSS, 1987).

Según UNECE-FAO EFC (1985) existen 15.000 ha de bosques de Pino Oregón regularmente manejadas en Holanda. Para España citan volúmenes netos de $6.820 \mathrm{ml} \mathrm{m}^{3}$ con corteza. Además de los países mencionados se ha plantado con éxito en Polonia y Dinamarca.

En Australia existen plantaciones experimentales de Pino Oregón ubicadas en Nueva Gales del Sur y Tasmania.

Debido a la buena forma, sanidad y a la alta productividad esta especie es la más importante después de Pinus radiata en Nueva Zelandia. Sus plantaciones llegan a 60.000 ha y se encuentran al sur de los $38^{\circ}$ LS (JAMES y BUNN, 1978; SUTTON, 1985).

En Latinoamérica la especie ha crecido con mayor éxito en Chile (BUCAREY, 1963). A Diciembre de 1987 las plantaciones cubrían 11.022 ha. ubicadas entre la VIII y XI Regiones (CORFO - INFOR, 1988). Las repoblaciones de mayor importancia se encuentran en el fundo "Voipir" en la localidad de Villarrica (X Región). 


\section{REFERENCIAS BIBLIOGRAFICAS}

1. BAILEY, L.H. 1960. The cultivated conifers in North America comprising the pine family and the taxads. New York, The MacMillan Co. 404 p.

2. BROCKMANN, C.F. 1979. Trees of North America. A guide to field identification. New York, Golden Press. 280 p.

3. BUCAREY, J. 1963. Pseudotsuga taxifolia (Poir.) Britt. en trumaos del cerro Voipir (Villarrica). Valdivia, Universidad Austral de Chile, Fac. Ing. For. 101 p.

4. BURSCHEL, P. und J. HUSS. 1987. Grundriss des Waldbaus. Berlin, Paul Parey Verlag. Parey's Studientexte $49.352 \mathrm{p}$.

5. CORFO - INFOR. 1988. Estadísticas forestales 1987. Boletín estadístico $\mathrm{N}^{\circ}$ 6. Santiago, CORFO INFOR. $10 \mathrm{p}$.

6. DALLIMORE, W. and A.B. JACKSON. 1961. A handbook of Coniferae, including Gingkoaceae. London, Edward Arnold Ltd. 686 p.

7. FOERST, K. 1980. Standort, Wuchsleistung und Ernährungszustand älterer bayerischer Bestände der grünen Douglasie (Pseudotsuga menziesil (Mirb.) Franco var. menziesii). Mitteilungen aus der Staatsforstverwaltung Bayerns, Heft $41.256 \mathrm{p}$.

8. FOWELLS, H.A. 1965. Silvics of forest trees of the United States. U.S.D.A., Agric. Handb. $N^{\circ} 217$. $762 \mathrm{p}$.

9. HERMANN, R.K. 1982. The genus Pseudotsuga: Historical records and past nomenclature. For. Res. Lab., Oregon State University, Corvallis. Special Publication 2A. 29 p.

10. - - - 1985. The genus Pseudotsuga: ancestral history and past distribution. For. Res. Lab., Oregon State University, Corvallis. Special Publication 2B. 32 p.

11. HOSIE, R.C. 1969. Nativa trees of Canada. Can. For. Serv. Ottawa, Queen's Printer. 380 p.

12. ISAAC, L.A. and E.J. DIMOCK, II. 1958. Silvical characteristics of Douglas-fir var.menziesii. U.S.D.A., For. Serv., PNW Silvical Series $\mathrm{N}^{\circ} 9$.

13. JAMES, R.N. and E.H. BUNN (eds.). 1978. A review of Douglas-fir in New Zealand. 16-19 September, 1974. FRI Symposium $\mathrm{N}^{\circ} 15.455 \mathrm{p}$.

14. LITTLE, E.L. Jr. 1979. Important forest trees of the United States. U.S.D.A. For. Serv., Agric. Handb. $\mathrm{N}^{\circ} 519.70 \mathrm{p}$.

15. OSWALD, H. et J. PARDE. 1984. Le Douglase en Françe: Sylviculture et production. Revue Forestière Francaise 36 (Special issue): 56-68.

16. RODRIGUEZ, G. y R. RODRIGUEZ. 1981. Las especies de Pinaceae cultivadas en Chile. Bosque 4 (1): 25-43.

17. SCHULLI, L. 1984. Le Douglase entre L'Haute-Rhin et le lac de Constance: 1'éxpérience acquise. Revue Forestière Française 36 (Special issue): 50-55.

18. STREETS, R.J. 1962. Exotic forest trees in the British Commonwealth. Oxford, Clarendan Press. 750 p.

19. SUTTON, W.R.J. 1978. A selective review of Douglas-fir in North America. FRI Symposium $\mathbf{N}^{\circ} 15$ : $310-322$.

20. - - 1985. Manejo de plantaciones de pino insigne. Transcripción de conferencia en Concepción y Santiago. Mimeografiado. Forestal Mininco S.A. 120 p.

21. U.N.E.C.E. and F.A.O. E.F.C. 1985. The forest resources of the E.C.E. region (Europe, URRS, NA). United Nations, New York. 233 p.

22. U.S.D.A. FOREST SERVICE. 1982. An analysis of the timber situation in the U.S. 1952-2030. Washington D.C., Forest Resource Report N ${ }^{\circ} 23.499$ p.

23. U.S. FOREST PRODUCTS LABORATORY. 1974. Wood handbook: Wood as an engineering material. U.S.D.A, Agric. Handb. $N^{\circ} 72.410$ p. 